\title{
Evidence for Linear Extrachromosomal Elements Mediating Gene Amplification in the Multidrug-Resistant J774.2 Murine Cell Line
}

\author{
Eckart U. Meese, Susan Band Horwitz, and Jeffrey M. Trent
}

\begin{abstract}
Previous studies from our laboratory have demonstrated specific cytogenetic alterations accompanying development of colchicine resistance in the 7774.2 murine cell line and in two sublines (J7.Cl-30 and J7.Cl-100) [1]. Although gene amplification is not observed in the parental J774.2 cell line, $a \sim 35$-fold amplification of the gene for p-glycoprotein (mdr) was noted in the 77 .Cl-30 subline (770-fold $\left.C L C^{H}\right)$ and $a \sim 70$-fold amplification in the $77 . C l-100$ subline (2500-fold $\left.C L C^{H}\right)$. In this study, we analyzed the localization and organization of the mdr gene. In the colchicine-resistant $\left(\mathrm{CLC}^{\mathrm{R}}\right)$ J7.Cl-30 subline, the p-glycoprotein domain was observed to reside on differently sized extrachromosomal elements. Our results indicate not only circular extrachromosomal elements but also linear extrachromosomal elements. By means of pulsed-field gel electrophoresis ( $P F G E$ ), the sizes of the extrachromosomal elements were shown to be $>2,500$ kilobasepairs (kb), $800 \mathrm{~kb}$, and $400 \mathrm{~kb}$. In contrast, the $77 . \mathrm{Cl}-100$ subline was characterized by the presence of homogeneously staining regions (HSRs). We have noted that with increasing colchicine resistance the extrachromosomal elements are replaced by HSRs. Our findings of linear elements that appear to be precursors of HSRs may offer a new way to interpret different theories of extrachromosomal gene amplification. The 77.Cl-30 cell line presents a unique system to analyze further the formation and structure of extrachromosomal elements.
\end{abstract}

\section{INTRODUCTION}

DNA amplification is known to occur in developmentally regulated genes [2]. Strong evidence also shows that amplified cellular oncogenes play a causative role in tumor initiation, progression, or both $[3,4]$. Furthermore, gene amplification is frequently associated with acquisition of the multidrug-resistant (MDR) phenotype [5, 6]. Amplified sequences have been localized in double minutes (dmin) and homogeneously staining regions (HSRs) $[7,8]$.

Despite the importance of dmins and HSRs to the process of gene amplification, little is known about the underlying molecular mechanism of their formation. Studies to date indicate that the structure of amplified DNA domains can be generated by' a variety of mechanisms and that the detailed events may vary in different cell types. In past years, several models for the generation of extrachromosomal elements and HSRs have been proposed. Recent models hypothesize a mechanism involving multiple replications at a single origin, leading to an "onion skin"-like structure $[4,9]$. Multiple initiations of replication have been noted in amplification events of chorion genes in

From the Department of Radiation Oncology. University of Michigan Medical Center, Ann Arbor, Michigan.

Address requests to: Eckart Meese, Ph.D., University of Michigan Medical Center, Department of Radiation Oncology. MSRBII C560B, 1150 W'. Medical Center Drive, Ann Arbor. MI 48109-0668.

Received May 1, 1991; accepted August 6, 1991.
Drosophila [10]. Schimke et al, suggested that perturbation of cell cycle patterns may result in chromosome fragmentation and generation of various recombination events [11]. Sen et al. suggested that prematurely condensed $S$-phase chromosomes are precursors of extrachromosomal elements |12|. Chromosomal regions that are prematurely' condensed while undergoing DNA replication are believed to be liberated from chromosomes, eventually forming HSRs. Based on this model, large linear DNA structures may be precursors for dmins. Wahl et al. proposed that dmins stem from smaller circular extrachromosomal elements [13|. These investigators showed that formation of submicroscopic extrachromosomal elements represents one of the earliest events in gene amplification. These small extrachromosomal elements are believed to enlarge in time to the size of microscopically detectable bodies (dmin), which eventually integrate into chromosomes to generate HSRs.

With the J774.2 cell line, we identified a unique system to analyze further the underlying mechanisms that lead to formation of extrachromosomal elements and HSRs. Based on our recent studies of mdr gene amplification in murine J7.Cl-30 cell lines, we analyzed the structure and location of the amplified DNA domain. In the (CLC $\left.{ }^{R}\right)$ J7.Cl-30 subline, we identified several extrachromosomal elements of different sizes. All the extrachromosomal elements have been shown to contain the amplified p-glycoprotein domain. Our study of the J7.Cl-30 cell line suggests that not

20 
only circular but also linear structures form a subset of the smaller extrachromosomal elements. Linear extrachromosomal elements of $42(\mathrm{~kb})$ kilobases long have previously been reported in yeast [14]. Circumstantial evidence also indicates linear dmins in neuroblastoma cells [15]. Because linear molecules may simply represent linearization of native DNA circles, we included several control experiments. First, we demonstrated that no significant breakage is caused by DNA preparation. Second, we excluded the possibility that cell density accounts for the linear extrachromosomal elements. Third, the presence of large extrachromosomal structures that appear to be intact in the nonirradiated cells was used as an internal control for random breakage.

Our study of the J7.Cl-100 subline indicates that the extrachromosomal elements are replaced by HSRs as the cells become more colchicine resistant. This aspect of our results is in good agreement with Wahl's model showing that smaller extrachromosomal elements are precursors of dmin which in turn can integrate into chromosomes to form HSRs. The presence of linear extrachromosomal elements in the J7.Cl-30 cell line can more easily be explained by alternative models, however (e.g., prematurely condensed chromosomes). Thus, our results indicate a novel mechanism that combines different aspects of recently proposed models for extrachromosomal gene amplification.

\section{MATERIALS AND METHODS}

\section{Cell Culture}

The parental J774.2 cell line and the colchicine-resistant sublines (J7.Cl-30 and $\mathrm{J} 7 . \mathrm{Cl}-100$ ) were maintained as previously described $[16,17]$. The colchicine-resistant sublines were derived by a stepwise increase of colchicine concentration. All cell lines were tested for mycoplasmal contamination.

\section{Cytogenetic Analysis}

Cells in exponential growth were harvested after 1 hour of colchicine treatment. Chromosomes were prepared as previously described [18].

\section{DNA Preparation, Gel Electrophoresis, and Southern Hybridization}

Cells were embedded in agarose plugs to a final concentration of $5 \times 10^{6} \mathrm{cells} / \mathrm{ml}$. DNA was prepared essentially as previously described [19]. To linearize extrachromosomal DNA circles, agarose plugs were placed in microcentrifuge tubes and exposed to gamma irradiation $\left({ }^{137} \mathrm{Cs}\right.$ source). Pulsed-field gel electrophoresis (PFGE) was performed using a modified contour clamped homogenous electric field (CHEF) system [20]. Gels (0.8\% agarose) were run in $0.5 \times$ TBE buffer (0.045 M Tris-borate, $0.001 \mathrm{M}$ EDTA) at $12^{\circ}-$ $14^{\circ} \mathrm{C}$ with varying switching times. Transfer and hybridization have been previously described [21]. DNA probes were labeled with ${ }^{32} \mathrm{P}$ by the random primer method of Feinberg and Vogelstein [22]. The probes used for this study were pgp1 and pgp2 (provided by V. Ling, Ontario Cancer Center Institute, Toronto, Canada) which hybridize to mdr1a and mdr1b, respectively $[23-25]$. After hybridization, the filters (zeta probe) were washed twice in $2 \times$ SSC/0.1\% sodium dodecyl sulfate (SDS) for 20 minutes each at room temperature, and twice in $0.1 \times \mathrm{SSC} / 0.1 \%$ SDS for 30 minutes each at $60^{\circ} \mathrm{C}$.

\section{RESULTS}

\section{Identification of Extrachromosomal Elements}

Molecular and cytogenetic analysis on J774.2, a mouse macrophage-like cell line, and two CLC ${ }^{R}$ sublines was performed to determine the organization of the amplified $p$ glycoprotein domain. The J7.Cl-30 subline, which exhibits a 35-fold mdr amplification was analyzed for the presence of extrachromosomal DNA. When metaphase spreads from the J7.Cl-30 subline were analyzed, extrachromosomal structures were observed in only a few metaphases spreads. Our cytogenetic analysis showed not only dmin bodies, but also single minutes in the J7.Cl-30 cells. In addition, the J7.Cl-30 cell was screened with PFGE for the presence of extrachromosomal elements. DNA was deproteinated in agarose plugs, separated by PFGE, and hybridized with pgp1 and pgp2 which identify the mouse mdr1a and $m d r 1 b$ genes, respectively. After hybridization of nonirradiated cells, a strong signal at $800 \mathrm{~kb}$ and a weaker signal at $400 \mathrm{~kb}$ were observed, indicating linear extrachromosomal DNA (Fig. 1A). After irradiation with $6 \times 10^{2}$ rad, a signal $>2,500 \mathrm{~kb}$ was detected in addition to the signals at 800 and $400 \mathrm{~kb}$. This signal at $>2,500 \mathrm{~kb}$ most likely represents DNA from the dmin identified in the metaphase spread. No difference was noted between probes pgp1 and pgp2. Our results indicate extrachromosomal structures of different sizes containing amplified p-glycoprotein domains in the J7.Cl-30 cell.

In the J7.Cl-100 cell line that exhibits a $\sim 70$-fold $\mathrm{mdr}$ amplification, PFGE analysis did not indicate the presence of extrachromosomal DNA. After hybridization with pgp1 and pgp2, a "smear," but no discrete band, was noted in the PFGE blot (Fig. 1C). These results are consistent with the karyotypic analysis. As previously described, HSR were identified in most cases and localized to a derivative 7 chromosome [der (7HSR)]. Hence, the dmin and the small extrachromosomal elements observed in the J7.Cl-30 subline ( $\sim 35$-fold amplified) are replaced by HSR in the J7.Cl-100 subline ( $\sim 70$-fold amplified). The replacement of extrachromosomal elements by HSR is consistent with the notion that dmin and smaller extrachromosomal element formation is an early event in the chronology of gene amplification, whereas formation of HSR represents a later event.

\section{Structural Analysis of Extrachromosomal Elements}

To analyze the structure of extrachromosomal elements in the J7.Cl-30 cell line, exposed the cells to gradually increasing gamma irradiation. Although the size of the $800-\mathrm{kb}$ signal was not affected by gamma radiation of up to $75 \times 10^{2}$ rad, there was an increase in signal intensity at low radiation doses (Fig. 2). This increase was observed consistently when J7.Cl-30 cells were exposed to low doses of gamma irradiation. These findings indicate circu- 


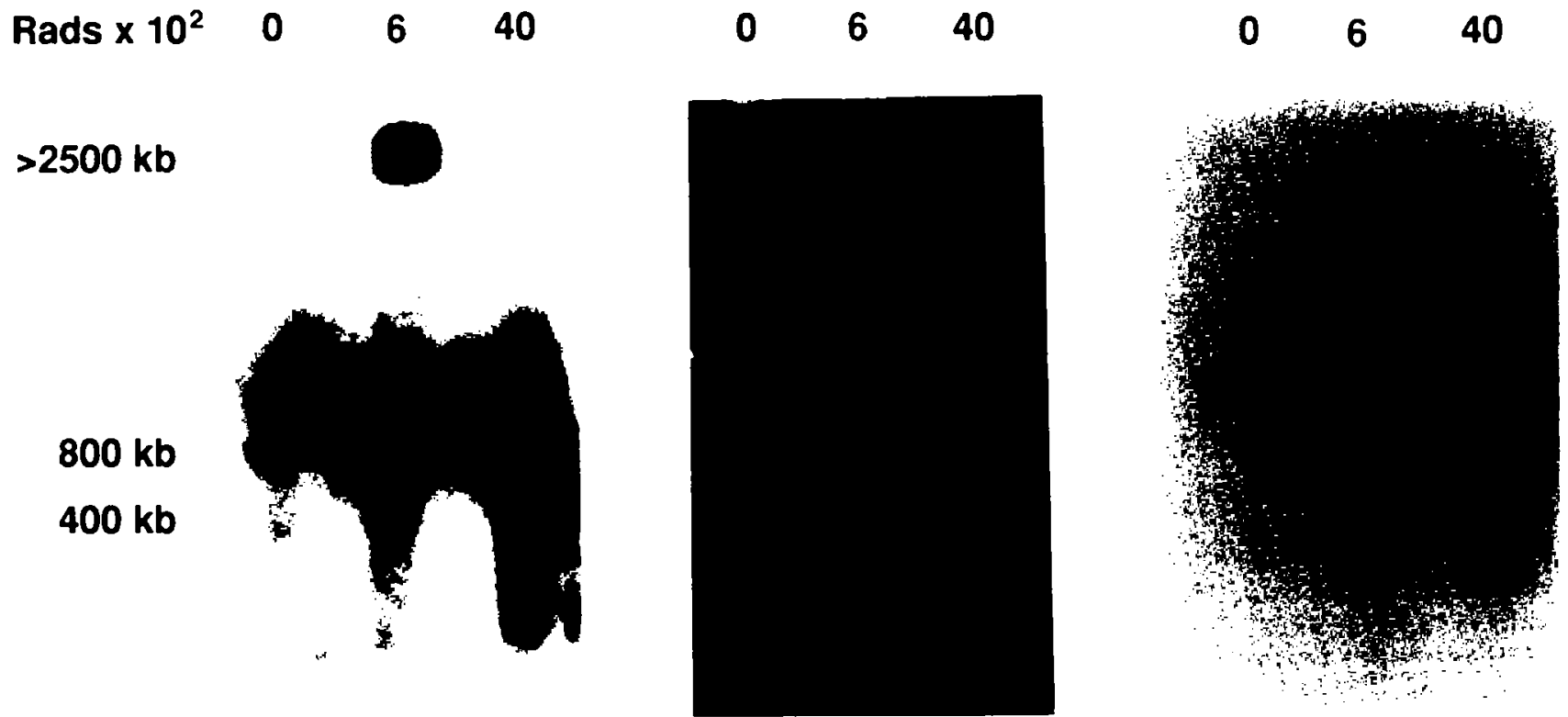

A

B

C

Figure 1 (A) Pulsed-field gel electrophoresis (PFGE) analysis of extrachromosomal elements in the J7.Cl-30 cell line. High-molecular-weight DNA was gamma-irradiated as indicated. size-fractionated by PFGE. and subsequently hybridized with the pgp1 probe. The blots were exposed for 8 hours. The running conditions were as follows: $125 \mathrm{~V}$. 180 -second pulse time, 28 -hour run time, temperature $14^{\circ} \mathrm{C}, 0.8 \%$ agarose in $0.5 \mathrm{TBE}$. The size of the fragments were estimated with yeast chromosomes (Beckman). After gamma irradiation at 600 rad, a hybridization signal at $>2,500$ $\mathrm{kb}$ is evident. The exposure time was 4 days to identify the $400-\mathrm{kb}$ signal. (B) Ethidium bromide stain of the PFGE gel. DNA was gamma-irradiated as indicated and size-fractionated. The running conditions were as in $A$. (C) Analysis by PFGE of amplified p-glycoprotein domains in the J7.Cl-100 cell line. INA from the Cl-100 subline was gamma-irradiated as indicated, size-fractionated by PFGE, transferred, and hybridized with pgp1. The running conditions were as in A. In contrast to the J7.Cl-30 subline, no extrachromosomal elements were identified.

Figure 2 PFCFE analysis of extrachromosomal elements in the 17.Cl-30 cell line. D.NA was gamma irradiated as indicated, fractionated by PFCE, transferred and probed with pgp 1 . The running conditions were as described in the legend to Fig. $1 \mathrm{~A}$. The strongest signal was noted at 2,000 rad of gamma irradiation. The increase in signal intensity at low irradiation doses indicates circular elements that are linearized by irradiation. After a dose of $15,000 \mathrm{rad}$, the signal at $800 \mathrm{~kb}$ was lost due to fragmentation.

\section{$\begin{array}{lllllll}\operatorname{Rads} \times 10^{2} & 0 & 20 & 40 & 60 & 75 & 150\end{array}$}

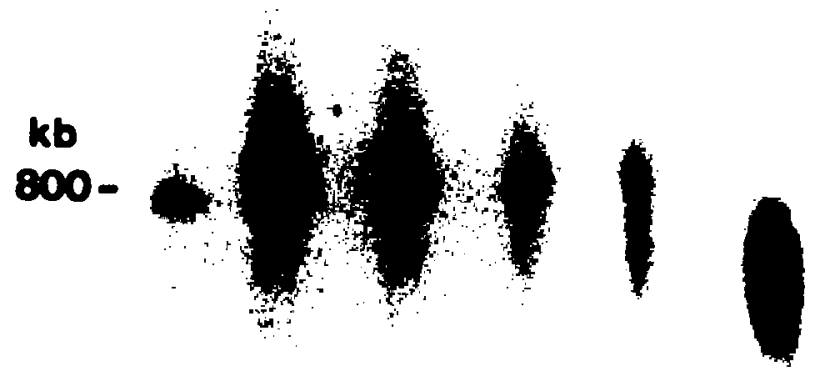




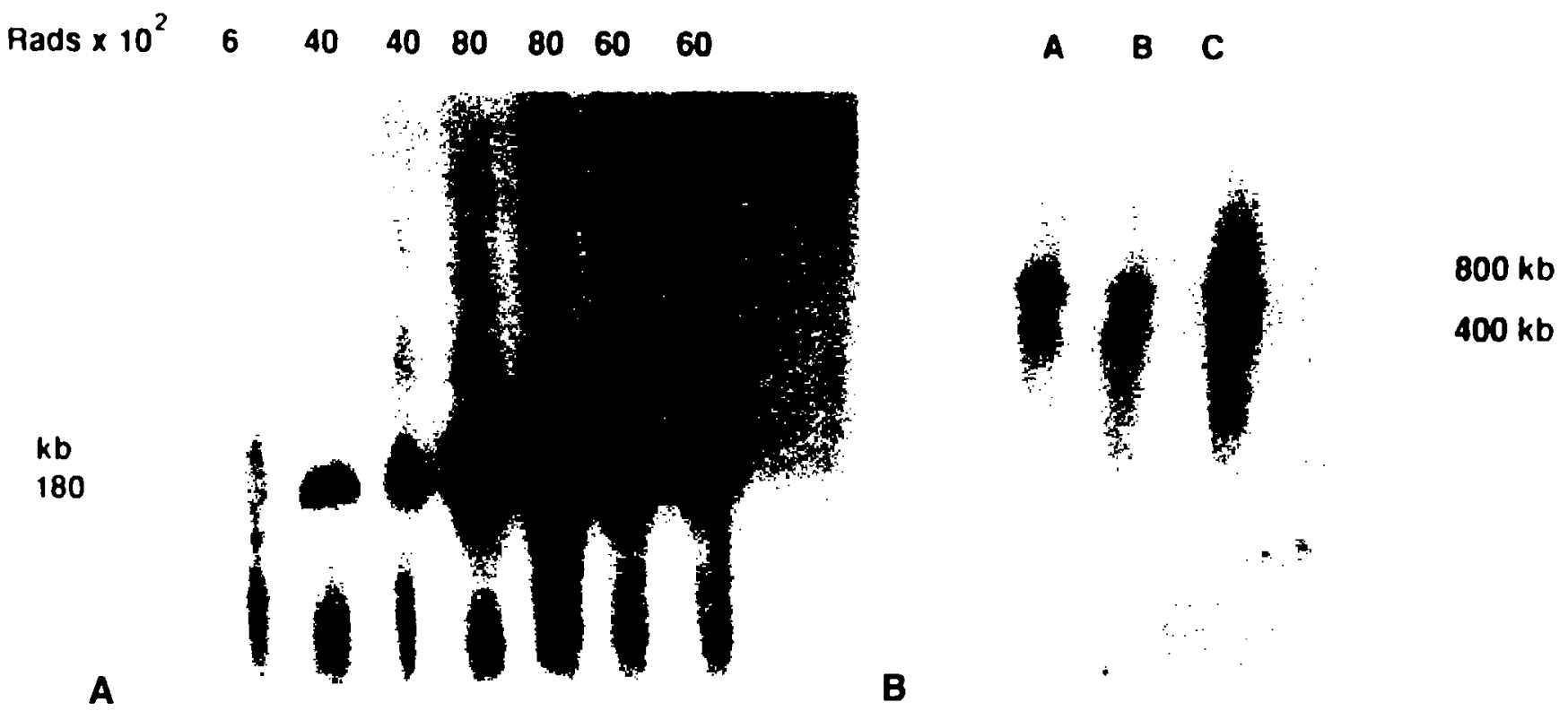

Figure 3 (A) Pulsed-field gel electrophoresis (PFGE) analysis of extrachromosomal circular elements in Raji cells. DNA was isolated from Raji cells containing 180-kb Epstein-Barr virus (EBV) episomes. After gamma irradiation of 0 to $80,000 \mathrm{rad}$, the DNA was fractionated by PFGE, transferred, and hybridized with an EBV-specific probe. The following running conditions were used: $120 \mathrm{~V}, 60$-second pulse time, and 40-hour run time. The other parameters were as indicated in the legend to Fig. 1A. No discrete band was noted in nonirradiated cells and in cells irradiated with 6,000 rad, whereas a strong signal at $180-\mathrm{kb}$ was detected when I.NA was exposed to higher doses of gamma irradiation. (B) Analysis by PFGE of extrachromosomal elements in J7.Cl-30 cells. Cells were harvested at different levels of confluencies. Lane A: Cells were grown to approximately $20 \%$ confluency (1 day). Lane B: Cells were grown to $100 \%$ confluency (2 days). Lane C: Cells were grown for 2 days beyond confluency. After size fractionation, the DNA was transferred and probed with pgp1. The running conditions were as described in the legend to Fig. $1 \mathrm{~A}$.

lar elements that are linearized by irradiation, thereby causing the increase in intensity of the $800-\mathrm{kb}$ signal. Hence, our results indicate circular fragments in the J7.Cl30 cell line.

As shown in Fig. 2, however, PFGE analysis also disclosed apparently linear extrachromosomal DNA in nonirradiated J7.Cl-30 cells. The finding of extrachromosomal elements migrating as linear molecules is at variance with Wahl's model of gene amplification which postulates only circular elements as dmin precursors. To analyze the structure of these smaller extrachromosomal elements further, we performed the following experiments.

First, circular molecules may be linearized in the process of DNA preparation and during gel electrophoresis. To exclude this possibility, we stained nonirradiated DNA by ethidium bromide after separation by PFGE (Fig. 1B). The ethidium bromide stain of the total DNA did not indicate disproportionally high levels of randomly broken DNA that could account for the hybridization signals at 800 and $400 \mathrm{~kb}$. In addition, using the same procedure as for the preparation of J7.Cl-30 DNA, we isolated DNA from Raji cells, which contain circular 180 -kb episomes. After hybridization with an Epstein-Barr virus (EBV)-specific probe, we noted no discrete band in nonirradiated cells, indicating intact DNA circles that do not enter the PFGE gel (Fig. 3A). As a positive control, DNA from Raji cells was exposed to increasing doses of gamma irradiation. Af- ter irradiation, a signal at $180 \mathrm{~kb}$ was detected, indicating linearized DNA molecules. These results demonstrate that no significant DNA breakage results from DNA preparation or fractionation.

Second, linearization of circular DNA molecules may occur during cell culture, e.g., due to cell death. To test the influence of cell density on linearization of DNA circles, harvested cells at different confluencies. We also included an experiment in which cells were grown for several days beyond confluency. In none of the experiments was the hybridization signal significantly altered, indicating that cell density did not cause significant DNA breakage of extrachromosomal elements in the J7.Cl-30 cell line (Fig. 3B).

Third, the simultaneous presence of extrachromosomal structures of different size offers a unique internal control for random breakage. Large circles are believed to be more susceptible to breakage than smaller extrachromosomal circles, but we never observed linearization of the large DNA circles of $\geq 2,500 \mathrm{~kb}$ in nonirradiated cells. These results indicate that random DNA breakage is unlikely to cause the linear DNA fraction at $800 \mathrm{~kb}$ observed in nonirradiated cells. Consequently, the signal in nonirradiated cells strongly indicates native linear fragments. This internal control is even more conclusive in light of the likely sequence similarity between dmin and smaller extrachromosomal structures. 


\section{DISCUSSION}

We identified linear extrachromosomal elements that contain an amplified mdr domain. Recent studies show the presence of linear plasmid-like structures in yeast and linear dmin in neuroblastoma cells $[14,15]$. According to our findings, large circles such as dmin might be formed by polymerization of varying numbers of linear elements. Such a concept could readily explain the size heterogeneity common in populations of extrachromosomal elements. Surprisingly, the linear elements appeared to be relatively stable as long as the J7.Cl-30 cells were grown in the presence of colchicine (data not shown). This stability might be conferred by telomeric sequences since they have been demonstrated in linear extrachromosomal elements in yeast [26]. Because telomeric sequences have been shown to be rich in origins of replication, telomeric regions could account for the autonomous replication of linear elements. Recent studies demonstrated a mechanism for the de novo synthesis of telomeric sequences [27].

Furthermore, our study provides further evidence that the small extrachromosomal elements are precursors of dmin, which in turn can integrate into chromosomal DNA from HSR. Our findings are consistent with results of several other studies of extrachromosomal elements and HSR. Specifically, using the CAD gene, Carroll et al. demonstrated that dmins can be produced from smaller precursors which were derived from small chromosomal deletions [28]. Further evidence that small extrachromosomal elements are involved in the formation of dmin was provided by Von Hoff et al. who described the formation of dmin from smaller elements in human leukemia cells [29]. In addition, in several human cell lines, amplified genes have been localized on extrachromosomal structures [30, 31]. Numerous examples also show that the location of the amplified domain differs from the location of the amplified genes in nonamplified cells [5, 32]. These findings, together with the notion that HSR can be formed at multiple chromosomal sites, supports the idea that dmin are precursors of HSR. Additional experimental evidence for the integration of dmins into chromosomes was provided by a recent study that demonstrated the shift of c-myc sequences from dmin to a chromosomal site [33], but circumstantial evidence also indicates that HSR can be generated de novo [34].

In this analysis, we present a unique system that offers further insight into the molecular mechanism leading to extrachromosomal structures. In part, the findings are consistent with the amplification model outlined above which postulates that HSR are derived from dmin and smaller extrachromosomal elements. The finding of linear structures can be more readily interpreted in light of alternative theories, however. Findings that prematurely condensed chromosomal regions can be precursors for extrachromosomal elements are in good agreement with the idea of large linear molecules being liberated from chromosomes to form dmin. Linear structures may represent early intermediates of extrachromosomal elements that are released from the genomic DNA by an excision or recombination mechanism.
The authors thank Sharon Olson for technical assistance and Barbara Burmeister for excellent secretarial assistance. They also thank Drs. D. VanDevanter and Paul Meltzer for helpful and critical discussion of the manuscript.

This work was supported in part by Grants No. CA-41183 and CA-39821 to (S.B.A.).

\section{REFERENCES}

1. Slovak ML, Lothstein L. Horwitz SB, Trent JM (1988): Molecular/cytogenetic alterations accompanying the development of multidrug resistance in the $\mathrm{J774.2}$ murine cell line. Leukemia 2:453-458.

2. Spradling AC, Mahowald AP (1980): Amplification of genes for chorion proteins during oogenesis in Drosophila melano. gaster. Proc. Natl Acad Sci USA 77:1096-2022.

3. Stark (GR (1986): DNA amplification in drug resistant cells and in tumours. Cancer Surv 5:1-23.

4. Schimke RT (1984): Gene amplification in cultured animal cells. Cell 37:705-713.

5. Stark GR, Wahl GM (1984): Gene amplification. Annu Rev Biochem 53:447-491.

6. Van der Bliek AM, Baas F, Van der Velde-Koerts T, Biedler JL, Meyers MB, Ozols RF, Hamilton TC, Joenje H, and Borst P. (1988a): Genes amplified and overexpressed in human multidrug resistant cell lines. Cancer Res 48:5927-5932.

7. Cowell JK (1982): Double minutes and homogeneously staining regions: Gene amplification in mammalian cells. Annu Rev Genet 16:21-59.

8. Hamlin JL, Milbrandt JD, Heintz. NH, Azizkhan JC (1984): IJNA sequence amplification in mammalian cells. Int Rev Cyto 90:31-82.

9. Botchan MW. Topp W. Sambrook J (1979): Studies on SV40 excision from cellular chromosomes. Cold Spring Harbor Symp Quant Biol 43:709-719.

10. Orr-Weaver Tl, Spradling AC (1986): Drosophila chorion gene amplification requires an upstream region regulating 18 transcription. Mol Cell Biol 6:4624-4633.

11. Schimke RT. Sherwood SW, Hill AB, Johnston RN (1986): Overreplication and recombination of DNA in higher eukaryotes: Potential consequences and biological implications. Proc Natl Acad Sci USA 83:2157-2161.

12. Sen $S$, Hittelman $W N$. Teeter I.1), Tien Kuo M (1989): Model for the formation of double minutes from prematurely condensed chromosomes of replicating micronuclei in drugtreated Chinese hamster ovary cells undergoing DNA amplification. Cancer Res 49:6731-6737.

13. Wahl GM (1989): 'The importance of circular CNA in mammalian gene amplification. Cancer Res 46:1333-1340.

14. Walton JD, Paquin CE, Kaneko K, Williamson VM (1986): Resistance to antimycin $A$ in yeast by amplification of $\mathrm{ADH} 4$ on a linear, $42 \mathrm{~kb}$ palindromic: plasmid. Cell 46:857-863.

15. Chen TL, Manuelidis L (1989): Neuroblastoma double minutes isolated by pulsed-field gel electrophoresis without prior strand-cleaving treatments. Genomics 4:430-433.

16. Lothstein L. Horwitz SB (1986): Expression of phenotypic traits following modulation of colchicine resistance in $\mathrm{J744.2}$ cells. J Cell Physiol 127:253-260.

17. Roy SN, Horwitz SB (1985): A phosphoglycoprotein associated with taxol resistance in J774.2 cells. Cancer Res 43: 3856-3863.

18. Trent J, Thompson F: (1987) Methods for chromosome banding of human and experimental tumors in vitro. Methods Enzymol 151:267-279

19. Meese F, Meltzer P, Trent I (1989): Application of natural 
partial digests to pulsed-field gel analysis of the amplified MDR locus. Genomics 5:371-374.

20. Meese E, Meltzer PS (1990): A modified CHEF system for PFGE analysis. Technique J Methods Cell Mol Biol 2:36-42.

21. Meese F, Meltzer PS, Witkowski CM, Trent JF (1989): Molecular mapping of the oncogene MYB and rearrangements in malignant melanoma. Genes Chromosomes Cancer 1:88-94.

22. Feinberg A, Vogelstein B (1984): A technique for labelling DNA restriction fragments to a high specific: activity. Anal Biochem 137:66-67.

23. Gerlach JH, Endicott JA, Juranka PF, Henderson G. Sarangi $F$. Deuchars KL, Ling V (1986b): Homology between P-glycoprotein and a bacterial haemolysin transport protein suggests a model for multidrug resistance. Nature 324:485-489.

24. Endicott JA, Juranka PF, Sarangi F, Gerlach JH, Deuchars KL and Ling V (1987): Simultaneous expression of two P-glycoprotein genes in drug-sensitive Chinese hamster ovary cells. Mol Cell Biol 7:4075-4081.

25. Hsu SI-H, Lothestein L, and Horwitz SB (1989): Differential overexpression of three $\mathrm{mdr}$ gene family members in multidrug-resistant J744.2 mouse cells. J Biol Chem 264:1205312062.

26. Szostak JW, Blackburn EH (1982): Cloning yeast telomeres on linear plasmid vectors. Cell 29:245-255.

27. Biessman $H$, Mason JM, Ferry K, d'Hulst M, Valgeirsdottir $K$, Traverse KL, Pardue M-L, (1990): Addition of telomere-associated HeT DNA sequences "heals" broken chromosome ends in drosophila. Cell 61:663-673.
28. Carroll SM, DeRose ML, Gaudray P, Moore CM, NeedhamVandevanter DR, Von Hoff DD, Wahl GM (1988): Double minute chromosomes can be produced from precursors derived from a chromosomal deletion. Mol Cell Biol 8:1525-1533.

29. Von Hoff DD, Forseth B, Clare CN, Hansen KL, VanDevanter D (1990): Double minutes arise from circular extrachromosomal DNA intermediates which integrate into chromosomal sites in human HL-60 leukemia cells. J Clin Invest 85:1887-1895.

30. Maurer BJ, Lai E, Hamkalo BA, Hood L. Attardi G (1987): Novel submicroscopic extrachromosomal elements containing amplified genes in human cells. Nature 327:434-437.

31. Von Hoff DD, Needham-VanDevanter DR, Yucel J, Windle BF, Wahl GM (1988): Amplified human c-myc oncogenes localized to replicating submicroscopic circular DNA molecules. Proc Natl Acad Sci USA 85:4804-4808.

32. Alitalo K (1985): Amplification of cellular oncogenes in cancer cells. Trends Biochem Sci 10:194-197

33. Cherif I), Lavialle C, Modjtahedi N, Le Coniat M, Berger R. Brison $O$ (1989): Selection of cells with different chromosomal localizations of the amplified c-myc gene during in vivo and in vitro growth of the breast carcinoma cell line SW 613-S. Chromosome 97:327-333.

34. Hyrien O, Debatisse M, Buttin G, Robert de Saint Vincent B (1988): The multicopy appearance of a large inverted duplication and the sequence at the inversion joint suggest a new model for gene amplification. EMBO J 7:407-417. 